\title{
NOTES D'ACTUALITÉ
}

\section{Le télétravail et le cotravail (coworking) : enjeux socioterritoriaux dans la foulée de la pandémie de COVID-19}

\author{
Diane-Gabrielle Tremblay ${ }^{\mathrm{a}}$
}

Le télétravail a toujours beaucoup intéressé les salariés, mais il ne touchait qu'au maximum $15 \%$ de la maind'œuvre québécoise et canadienne en 2019. Par contre, depuis l'arrivée de la pandémie de COVID-19, le taux de télétravail a explosé, Statistique Canada l'ayant estimé à $40 \%$ en avril 2020. Des millions de télétravailleurs se sont ajoutés en l'espace de deux semaines!

Nécessité fait loi : des masses de personnes sont passées en télétravail, alors que les entreprises comme la fonction publique canadienne et québécoise ont tenté de ralentir les pertes d'emploi et la réduction des heures de travail. De fait, l'emploi a baissé de 5,3\% et les heures effectivement travaillées à l'emploi principal ont chuté de 15,1\% en mars 2020 (Statistique Canada, 2020). Les données d'avril devraient être pires. Par contre, le contexte actuel de confinement n'est pas toujours aussi facilitant pour le télétravail, car plusieurs mères (et pères) se voient contraints de faire du télétravail, tout en continuant de porter l'essentiel du fardeau familial (England, 2010; Moyser et Burlock, 2018; Tremblay, 2019, 2003a, 2003b, 2002). Sans oublier que, pendant la pandémie, les écoles et les garderies ont fermé et les enfants sont restés à la maison à temps plein pendant au moins deux mois. Enfin, il faut noter que bon nombre de travailleurs, dans les services de santé et autres services de proximité, ainsi que dans la fabrication manufacturière, n'ont pas accès au télétravail, de sorte que certains ne peuvent profiter de cette modalité de travail pour se protéger du virus.

Par ailleurs, des auteurs mettent en évidence le fait que le télétravail (Scaillerez et Tremblay, 2016; Tremblay, 2003a, 2003b) et le développement du coworking (Krauss et Tremblay, 2019) pourraient améliorer le dynamisme et le développement des régions, tout en offrant la perspective de réduire les déplacements et la consommation de pétrole, ainsi que la pollution qui s'ensuit. Il faut noter que, depuis quelques années, nombre d'organisations, publiques et privées, avaient commencé à réaménager leurs espaces de travail, développant des aires ouvertes, des bureaux partagés et du coworking, ce qui permet en parallèle le développement du télétravail pour les emplois exigeant plus de concentration. Si le mouvement était lent, il s'accélèrera certes dans la foulée de la pandémie et des enjeux de distanciation physique, qui persisteront dans les prochains mois et années. Certains travailleurs souhaiteront toutefois retourner au lieu de travail habituel, et plusieurs employeurs le souhaiteront également. Ainsi, s'il est certain que le télétravail sera une pratique courante dans les prochains mois et années, il y aura sans doute un recul par rapport à la période du confinement.

Les moments de crise sont souvent des moments de changement radical, et l'organisation du travail actuelle sera manifestement remise en question par le confinement et par la distanciation physique prescrits par la pandémie. Certains pensent que cette crise pourrait aussi avoir des effets sur les régions ainsi que sur le développement des banlieues des villes des régions plus éloignées. Si, dans les années passées, certains résidents des grandes régions de Montréal ou de Québec avaient commencé à travailler quelques jours par semaine dans une seconde résidence située en région, plusieurs pourraient l'envisager de manière

a Professeure, université TÉLUQ (Université du Québec), Directrice de l'ARUC sur la gestion des âges et des temps sociaux 
plus régulière et à plus long terme, si le télétravail devenait pratique courante dans leur organisation (Scaillerez et Tremblay, 2016). Un des principaux freins au télétravail est la résistance des superviseurs ou cadres intermédiaires. Or, la pandémie aura permis à nombre d'entre eux de l'expérimenter et de constater qu'il n'y a pas perte de productivité, comme ils pouvaient le craindre, et ce, même si certains ont plus de difficulté avec les enfants présents à la maison. Avec la réouverture des écoles et des garderies, le télétravail à partir de la maison sans la présence des enfants à domicile ne pourra qu'être plus productif.

Les résultats de recherche étaient déjà clairs, mais les préjugés persistaient, notamment l’idée selon laquelle les télétravailleurs pourraient faire diverses tâches domestiques et autres, ce qui les rendrait moins productifs au final. Pourtant, comme d'autres recherches menées dans des pays précurseurs en matière de télétravail (Suède, Pays-Bas, États-Unis), notre recherche menée avec le Centre facilitant la recherche et l'innovation dans les organisations (CEFRIO, 2001; Tremblay, 2001a, 2001b) avait déjà montré que la productivité et la qualité du travail étaient maintenues, voire améliorées. Plus récemment, une enquête d'Indeed (Wolfe, 2019), menée auprès de plus de 500 employés et 500 employeurs dans divers secteurs, a montré que $96 \%$ des organisations qui avaient une politique et pratique régulière du télétravail avaient constaté que le télétravail ne nuisait aucunement à la productivité, et $65 \%$ avaient même constaté une hausse de productivité.

Parmi les inconvénients du télétravail, on note un risque d'isolement et d'exclusion des décisions importantes dans l'organisation (Tremblay, 2013, 2006). Si le risque d'isolement des télétravailleurs est réel, plusieurs affirment qu'il est toujours possible d'être présents au bureau de temps en temps, et la pratique du télétravail en période de confinement au printemps 2020 a fait bondir le nombre de réunions en visioconférence sur des plateformes comme Zoom, Skype ou autres, de sorte que le risque d'invisibilité s'est beaucoup réduit.

Les années à venir verront certes progresser le télétravail, ce qui pourrait se traduire par une délocalisation vers les banlieues et les petites villes en région. Il faudra alors s'interroger sur l'arbitrage entre les effets positifs de la déconcentration (réduction de la pollution des voitures et des besoins en infrastructures comme les routes et les transports collectifs) et les effets négatifs (accroissement des besoins en infrastructures numériques et hausse des gaz à effet de serre associés à ce qu’on appelle la " pollution numérique »1).

Autre changement sur le plan territorial : la multiplication des espaces de travail et de coworking ou de cotravail au Québec depuis quelques années, et ce, non seulement dans les grandes villes comme Montréal et Québec, mais aussi dans les petites villes en région (Krauss et Tremblay, 2019; voir l'article de Tremblay et Vaineau dans ce numéro). Au moment de la « mise sur pause » de l'ensemble du Québec en mars 2020, plusieurs de ces espaces ont fermé ou ont vu leurs activités ralentir, car les travailleurs en coworking craignaient de travailler en espace ouvert, comme c'est plus souvent le cas dans les espaces de coworking. Par contre, notre dernière recherche (voir Krauss et Tremblay, 2019 et l'article dans ce numéro de $\mathrm{O}$ et T) sur les espaces de coworking en région montrait qu'on trouve aussi beaucoup de petits bureaux privés dans les espaces de coworking en région. On pourrait donc imaginer que certaines personnes choisiraient de s'établir en région, plus loin des bureaux de leur employeur, et que certaines choisiraient aussi de s'associer à un espace de coworking pour ne pas travailler de manière isolée à domicile et pour profiter des réseaux professionnels, installations informatiques, salles de réunion ou autres équipements que peuvent offrir les espaces de coworking. Ces espaces permettent de partager des infrastructures et certains les inscrivent directement dans l'économie « du partage », elle aussi perçue comme positive sur le plan environnemental.

Selon l'enquête Indeed précitée (Wolfe, 2019), de nombreuses personnes souhaitent s'établir à distance, en région. En effet, $60 \%$ des employés affirment que le télétravail constitue un critère important pour le choix d'un emploi, et même $36 \%$ des employés envisagent d'accepter un salaire inférieur contre la possibilité de travailler à distance. La pandémie ayant fait la preuve que les employés peuvent être aussi performants à distance et nombre d'entreprises cherchant à réduire leurs frais locatifs, on peut penser qu'il y aura progression du télétravail et du travail en espace de coworking. Pour éviter le sentiment d'isolement et pour assurer les communications avec l'employeur, il faudra prévoir étendre l'accès à Internet 
haute vitesse afin que les équipes travaillant sur des projets conjoints puissent échanger de l'information, se rencontrer « en ligne » et travailler sur des documents communs. Les gestionnaires devront aussi changer leur mode de gestion, passant du contrôle à l'encadrement, de la gestion «à vue » à la gestion par objectifs et résultats.

Bref, bien que le télétravail soit actuellement introduit en contexte de crise, et que la préparation n'ait pas été très longue dans la plupart des cas, il faut reconnaitre que certaines organisations fonctionnaient déjà avec une bonne dose de télétravail et que nombre de professionnels et de cadres, comme d'employés de bureau, sont en mesure de réaliser leur travail depuis leur domicile ou encore d'un espace de coworking. Ainsi, le télétravail et les espaces de coworking pourraient connaitre une progression au lendemain de la pandémie, car les préjugés sur le travail à distance seront tombés, l'expérience ayant montré qu'il pouvait au contraire fournir la performance attendue et même, dans le cas du coworking, des sources nouvelles de réseautage, d’innovation et de créativité (Krauss et Tremblay, 2019).

L'expérimentation à grande échelle que permet cette pandémie amènera certes un bon nombre d'organisations à maintenir la pratique du télétravail, même s'il faut aussi reconnaître que le télétravail ne peut s'appliquer à tous les types d'emplois, certains services (santé, restauration, etc.) notamment en étant exclus. On peut donc s'attendre à ce que le télétravail progresse dans plusieurs organisations après la période de pandémie. Déjà, nombre d'organisations prévoient prolonger le télétravail jusqu'en septembre, voire en décembre 2020. Cependant, on peut aussi penser que, puisque l'être humain est un « être social» et que la créativité et l'innovation sont souvent favorisées par la mise en commun d'idées et l'échange, il faudra s'assurer d'une bonne qualité et d'une grande capacité des services Internet haute vitesse. Selon les modalités de télétravail, à temps plein ou partiel, l'impact sur la délocalisation et la présence en région sera plus ou moins important, mais on peut certes prévoir des développements sur ce plan au cours des prochaines années.

\section{NOTE}

1 Selon un rapport diffusé par TV5, 'la pollution numérique consomme 10\% de l'électricité mondiale'. 'Ce n'est pas seulement l'utilisation des équipements qui pollue mais toutes les étapes du cycle de vie des objets numériques.' https://information.tv5monde.com/info/pollution-numerique-comment-reduire-ses-effets-au-quotidien-279020 (page consultée le 12 mai 2020)

\section{RÉFÉRENCES}

CEFRIO. (2001). Le télétravail. Montréal : IQ éditeur.

England, P. (2010). The gender revolution: Uneven and stalled. Gender \& Society, 24(2), 149-166. Repéré à https://doi.org/10.1177/0891243210361475

Krauss, G. et Tremblay, D.-G. (dir.). (2019). Tiers-lieux - travailler et entreprendre sur les territoires : espaces de coworking, fablabs, hacklabs. Rennes/Québec : Presses universitaires de Rennes/Presses universitaires du Québec.

Moyser, M. et Burlock, A. (2018). Emploi du temps : la charge de travail totale, le travail non rémunéré et les loisirs. Femmes au Canada : rapport statistique fondé sur le sexe, no $890503 \mathrm{X}$ au catalogue. Ottawa : Statistique Canada. Repéré à www150.statcan.gc.ca/n1/fr/pub/89-503-x/2015001/article/54931-fra.pdf?st=rfXwVUkz

Scaillerez, A. et Tremblay, D.-G. (2016). Le télétravail, comme nouveau mode de régulation de la flexibilisation et de l'organisation du travail : analyse et impact du cadre légal européen et nord-américain. Revue des organisations responsables, 11(1), 21-31. Repéré à https://doi.org/10.3917/ror.111.0021

Statistique Canada. (2020). Tableau de bord sur l'économie du Canada et la COVID-19. Ottawa : Statistique Canada. Repéré à www150.statcan.gc.ca/n1/pub/71-607-x/71-607-x2020009-fra.htm?HPA=1

Tremblay, D.-G. (2001a). Télétravail : articuler qualité de vie et performance (Rapport de recherche). Montréal : CEFRIO. Repéré à https://cefrio.qc.ca/media/1818/teletravail-articuler-qualite-de-vie-et-performance.pdf

Tremblay, D.-G. (2001b). Le télétravail : définitions et enjeux. Dans CEFRIO (dir.), Télétravail : concilier performance et qualité de vie (p. 23-32). Montréal : IQ éditeur et CEFRIO. 
Tremblay, D.-G. (2002). Balancing work and family with telework? Organizational issues and challenges for women and managers. Women in Management, 17(3/4), 157-170. Repéré à https://doi.org/10.1108/09649420210425309

Tremblay, D.-G. (2003a). Telework: A new mode of gendered segmentation? Results from a study in Canada. Canadian Journal of Communication, 28(4), 461-478. Repéré à https://doi.org/10.22230/cjc.2003v28n4a1393

Tremblay, D.-G. (2003b). Le télétravail : ses impacts sur l'organisation du travail des femmes et la conciliation emploi-famille (note de recherche no 2003-10). Montréal : TÉLUQ, Chaire du Canada sur les enjeux socio-organisationnels de l'économie du savoir. Repéré à www.teluq.ca/chaireecosavoir/pdf/NRC03-10.pdf

Tremblay, D.-G. (2006). Le télétravail. Dans J. Akoka et I. Comyn-Wattiau (dir.), Encyclopédie de linformatique et des systèmes d'information. Paris : Vuibert.

Tremblay, D.-G. (2013). Telework. Dans V. Smith (dir.), Sociology of work: An encyclopedia (p. 871-872). Thousand Oaks (CA) : SAGE.

Tremblay, D.-G. (2019). Conciliation emploi-famille et temps sociaux. Québec : Presses de l'Université du Québec.

Wolfe, P. (2019). Télétravail : $62 \%$ des employeurs canadiens permettent cette pratique. Infopresse, [Blogue]. Repéré à www.infopresse.com/article/2019/2/18/une-approche-equilibree-du-teletravail 